\title{
Assembleias de peixes associadas aos bancos de macrófitas aquáticas em lagos manejados da Amazônia Central, Amazonas, Brasil
}

\author{
Maria Gercilia Mota SOARES ${ }^{1, *}$, Carlos Edwar Carvalho FREITAS ${ }^{2}$, Ana Cristina Belarmino de OLIVEIRA ${ }^{2}$ \\ 1 Instituto Nacional de Pesquisas da Amazônia (INPA/CBI0). Av. André Araújo, 2936, Petrópolis, CEP: 69080-97. Manaus, Amazonas, Brasil. \\ * Autor Correspondente: gerciliams@yahoo.com.br \\ 2 Universidade Federal do Amazonas (UFAM/DEPESCA). Av. Gal. R. 0. Jordão, 3000, Coroado, CEP: 69077-000. Manaus, Amazonas, Brasil. \\ cefreitas@ufam.edu.br, acbolive@ufam.edu.br
}

\section{RESUMO}

O co-manejo dos recursos pesqueiros que vem sendo desenvolvido pelos ribeirinhos da Amazônia têm a preocupação de assegurar ambientes adequados para a conservação dos estoques. Esta estratégia de co-manejo é baseada em regras de acesso e uso dos recursos pesqueiros. Nesse estudo, foi investigada a influência do tipo de uso de lagos (preservados e manejados para subsistência) e a sua distância do rio (próximos e distantes) na estrutura das assembleias de peixes associadas aos bancos de macrófitas aquáticas em lagos de várzea, Amazônia Central. Os peixes foram capturados na cheia com rede de cerco em seis lagos com distância do rio variando de 0,87 a 10,9 km. Nas macrófitas aquáticas e capins flutuantes dos lagos foram capturados um total de 623 exemplares de peixes, distribuídos em 56 espécies. A análise de covariância (ANCOVA) indica que o co-manejo dos lagos e distância não influenciaram significativamente nos atributos ecológicos das assembleias (abundância, riqueza, peso total, diversidade de Shannon-Weaver, diversidade Berger-Parker, equitabilidade e dominância). A análise de similaridade (ANOSIM) também mostrou que não existe diferença na composição de espécies entre os tipos de lago. Estes resultados sugerem outros fatores, como o pouco tempo de manejo efetivo, a agricultura como sendo a principal atividade econômica de subsistência, inexistência de pescarias em larga escala que produzam alteraçóes ambientais significativas e a existência de um fator ecológico de grande intensidade, o pulso de inundação, sobrepondo a outros de menor intensidade.

PALAVRAS-CHAVE: Rio Amazonas, ictiofauna, lago de preservação, lago de subsistência.

\section{Fish assemblage associated with aquatic macrophytes bank in mananged lakes of Central Amazon, Amazonas, Brazil}

\section{ABSTRACT}

The co-management of fishery resources developed by Amazonian riverine people is concerned with the maintenance of suitable environments for fish stock conservation. This type of co-management strategy is based on rules of access and use for fishery resources. In this research we investigated the influence of using lakes (preserved and managed for subsistence) and its distance from the river (near and far) in the structure of the fish assemblages associated with floating meadows bank in floodplain lakes, Central Amazonian. Fish were caught during flood period using seine nets in six lakes in a distance ranging from 0.87 to $10.9 \mathrm{~km}$ from the river. In floating meadows bank were captured a total of 623 specimens of fish, over 56 species. The analysis of covariance (ANCOVA) indicates that the co-management of lakes and distance did not influence significantly the ecological attributes of fish assemblages (abundance, richness, total weight, Shannon-Weaver and Berger-Parker diversity, evenness and dominance). According to analysis of similarity (ANOSIM) there was no difference in species composition among the lakes. These results suggest other factors, such as: short time of effective management, the agriculture as the most important economic activity of subsistence, the lack of fisheries in large-scale which cause significant environmental changes and the existence of an ecological factor of great intensity, "the flood pulse", overlapping others of less intensity.

KEYWORDS: Amazon River, fish fauna, preservation lake, subsistence fishing lake. 


\section{INTRODUÇÃO}

$\mathrm{Na}$ Amazônia Central, os bancos de macrófitas aquáticas e capins flutuantes constituem um dos habitats de alta complexidade e de importância nos lagos de várzeas (Goulding 1997). A alta produtividade das plantas que constituem o habitat permite a existência de nichos ecológicos que abrigam muitos animais aquáticos, semi-aquáticos e terrestres (Junk 1973; Lopes et al. 2011). As assembleias de peixes associadas aos bancos de macrófitas possuem estrutura diferente de acordo com o tipo de estande, como os monoespecíficos de Eichhornia crassipes e E. azurea que podem influenciar a distribuição da ictiofauna (Sánchez-Botero et al. 2003). Os peixes também utilizam estes habitats como meio de dispersão (Schiesari et al. 2003; Bulla et al. 2011), refúgio e berçário (Sánchez-Botero e Araújo-Lima 2001) para os juvenis e espécies de pequeno porte.

No emaranhado de raízes das plantas dos bancos de macrófitas nos lagos de várzea já foram capturadas até 139 espécies de peixes (Petry et al. 2003). No lago Mamirauá (Henderson e Hamilton 1995) e nos lagos do trecho ManausCoari foram estimada a biomassa de peixes (Prado et al. 2010), nos lagos da ilha da Marchantaria, a riqueza de peixes está relacionada com o tipo de estande de macrófitas aquáticas (Petry et al. 2003).

As populaçóes ribeirinhas vêm desenvolvendo um regime de manejo de lagos com acordos onde incluem regras de acesso e de uso dos recursos pesqueiros. Uma regra relevante se refere ao estabelecimento de lagos que devem ser destinados para a pesca de subsistência, enquanto outros são mantidos sem exploração, destinados a contribuir para a conservação dos estoques pesqueiros. Isso é resultado da preocupação em estabelecer um manejo que permita a existência de ambientes favoráveis ao desenvolvimento dos peixes, uma vez que a manutenção e até mesmo a recuperação de populaçôes de peixes está relacionada, em parte, com a sobrevivência das larvas e dos jovens. Diante disso, considerando a importância dos habitats formados pelas macrófitas para os estágios iniciais de diversas espécies de peixes explotadas pela pesca comercial, este trabalho investiga a influência do tipo de uso de lagos (preservado e manejado para subsistência) e a sua distância do rio (próximos e distantes) na estrutura das assembleias de peixes associadas aos bancos de macrófitas aquáticas em lagos de várzea da Amazônia Central.

\section{MATERIAL E MÉTODOS}

\section{Área de estudo}

O trabalho foi realizado em seis lagos de várzea situados no rio Amazonas, nas proximidades da cidade de Itacoatiara, distante $270 \mathrm{~km}$ de Manaus. Quatro lagos estáo localizados na ilha do Risco, cerca de $15 \mathrm{~km}$ a jusante de Itacoatiara:

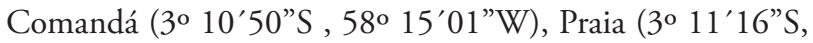
$58^{\circ} 16^{\prime} 57^{\prime \prime}$ W), Tracajá (30 09'51'S, 58 14'59”W) e Acari

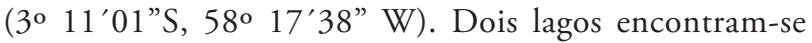
no Complexo Lacustre do rio Arari, na margem direita do rio Amazonas: Araçá (319'54”S e 58०18'25”W) e

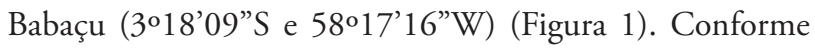
os critérios definidos pelos comunitários, os lagos próximos à área residencial, Praia (PRAm), Comandá (COMm), Araçá (ARAm), estão sendo manejados para subsistência (ou manutenção), consumo exclusivo da comunidade e os mais distantes e de difícil acesso, Acari (ACAp), Tracajá (TRAp) e Babaçu (BABp), são mantidos para preservação (ou procriaçáa), onde a pesca é proibida. Esses lagos estáo situados em diferentes posições do gradiente de inundação do rio Amazonas. Os mais próximos do rio estáo na ilha do Risco: Praia a 0,87 km, Comandá a 1,04 km, Acari a 1,71 $\mathrm{km}$, e o Tracajá situado a 2,27 km. O Praia e o Comandá estão conectados diretamente com o rio. Os mais distantes do rio estão no Complexo Lacustre do rio Arari: o Araçá localizado a 10,9 km e o Babaçu a $9 \mathrm{~km}$ (Figura 1).

\section{Coleta e análise dos dados}

Os peixes foram capturados nos bancos de macrófitas aquáticas de 6 lagos na época de cheia (junho/2002) utilizando redes de cerco medindo $20 \mathrm{~m}$ de comprimento x $5 \mathrm{~m}$ de altura e malha de $5 \mathrm{~mm}$ entre nós opostos. Em cada lago foi realizada uma amostragem. Após as capturas, os peixes foram etiquetados e fixados em formol a $10 \%$ e depois preservados em álcool a 70\%. A identificação dos peixes foi realizada com o auxílio de chaves sistemáticas (Géry 1977; Kullander 1986; Mago-Leccia 1994; Planquette et al. 1996) e de especialistas do Instituto Nacional de Pesquisas da Amazônia - INPA.

A caracterização dos bancos de macrófitas aquáticas em cada lago foi efetuada por demarcaçáo de cinco faixas de $1 \mathrm{~m}$ de largura ao longo de um transecto, que vai desde o limite da floresta inundada até a sua linha final de ocorrência, em direção da água livre (Rubin 2001). As faixas foram subdivididas em quadrados de $1 \mathrm{~m}$, e em cada quadrado foram registradas as espécies.

Os atributos ecológicos das assembleias de peixes foram analisados usando medidas de abundância absoluta $(\mathrm{N})$, do peso total $(\mathrm{Pt})$ em gramas $(\mathrm{g})$, da riqueza $(\mathrm{S})$, do índice de diversidade de Shannon-Weaver (H') (Krebs 1989) e do inverso do índice de dominância de Berger-Parker (1/d) (Berger e Parker 1970). Para complementar a interpretação do índice de Shannon-Weaver, foi calculado o índice de equitabilidade (E) (Krebs 1989). Além disso, a riqueza potencial de espécies foi estimada pela curva de rarefação (Krebs 1989) e pelo índice JackKnife 1 (Heltshe e Forrester 1983).

Para avaliar a influência do tipo de uso (manejado para subsistência e de preservaçáo) e distância dos lagos do rio 

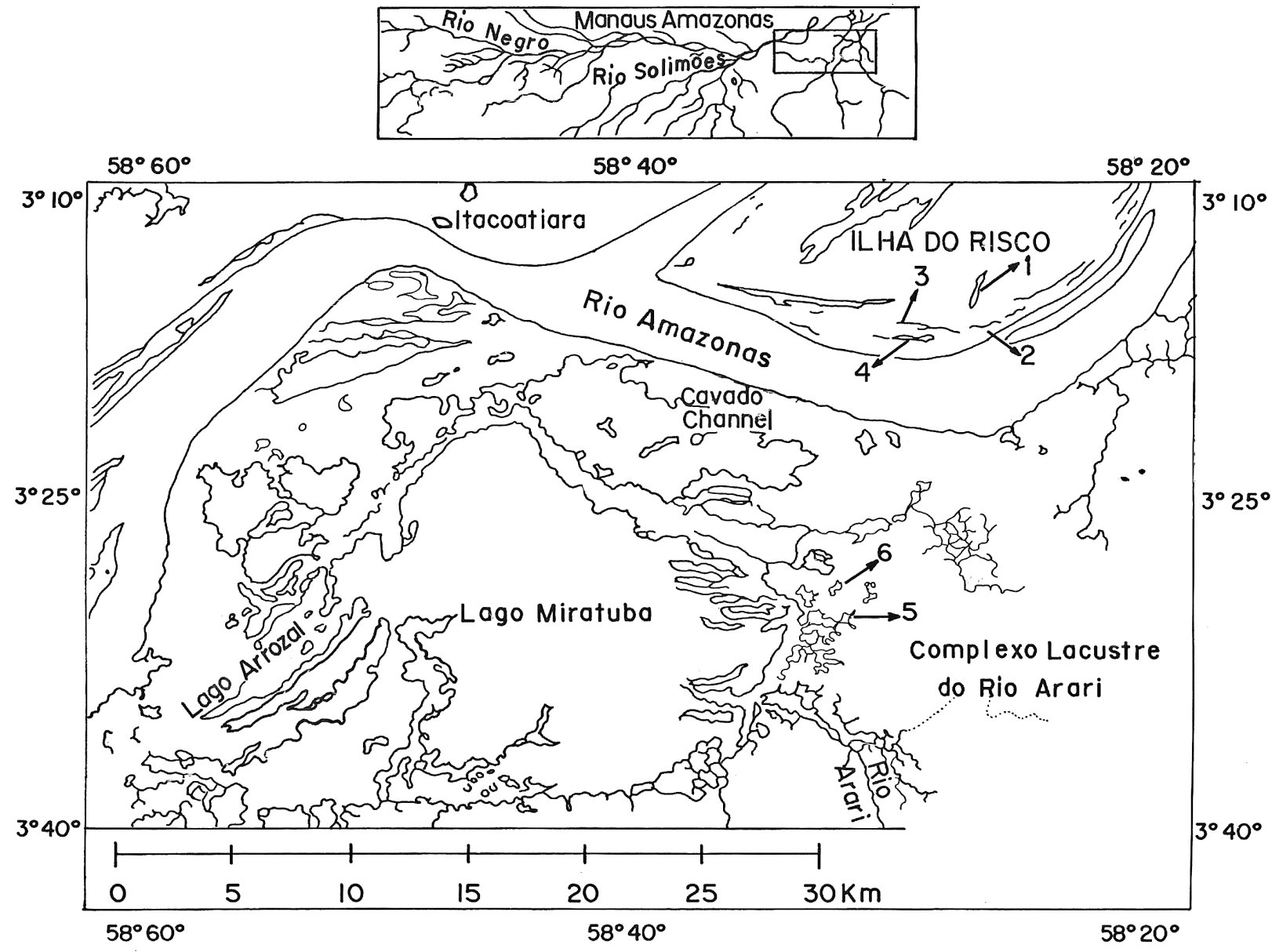

Figura 1 - Localização dos lagos na ilha do Risco e no Complexo Lacustre do rio Arari, Amazônia Central, AM. 1-Tracajá (preservação), 2-Comandá (manejo), 3-Acari (preservação), 4-Praia (manejo), 5-Araçá (manejo), 6-Babaçu (preservação).

(próximos e distantes) na estrutura das assembleias de peixes foi realizada uma análise de covariância (ANCOVA) (Zar 1996). Como variáveis resposta $(\mathrm{Y})$ foram utilizadas os atributos ecológicos das assembleias. Assim, temos diferentes ANCOVAS para cada Y considerado. Neste caso não é possível fazer uma MANCOVA porque as variáveis $Y$ não são estocasticamente independentes, visto que foram calculadas utilizando o mesmo conjunto de dados. Os pressupostos da ANCOVA foram atendidos: a independencia entre o tratamento e a covariante (homogeneidade das linhas de regressão), e, mensuraçáo do covariante sem erro.

$\mathrm{Na}$ primeira análise, as variáveis foram utilizadas em conjunto de acordo com o seguinte modelo:

$Y=\alpha+\beta 1 *$ Tipo de uso dos lagos + Distância dos lagos; (1)
Onde:

As variáveis resposta são a abundância, riqueza, peso total, diversidade de Shannon-Weaver, diversidade Berger-Parker, equitabilidade e dominância e as indicadoras são o tipo de uso dos lago e distância do lago em relação ao rio. Cada variável resposta foi analisada separadamente no modelo acima descrito. As análises foram realizadas utilizando o pacote de software comercial Statistica 6.0.

Para verificar se existe diferença significativa na composiçáo de espécies entre os tipos de lagos, foi realizada uma análise de similaridade (ANOSIM), um teste de permutaçáo baseado nas similaridades das amostras (Clarke 1993) utilizando o programa estatístico R 2.15 (R Development Core Team 2012). Graficamente é ilustrado por uma análise de cluster UPGMA usando dados quantitativos (Bray-Curtis) com o programa Past v. 2.17b. 


\section{RESULTADOS}

$\mathrm{Na}$ cheia, todos os lagos estavam margeados por extensos bancos de macrófitas aquáticas e capins flutuantes, tendo sido identificadas um total de 17 espécies com riqueza variando entre 5 e 10 espécies. As macrófitas flutuantes livres predominaram (13 espécies), seguida pelas emergentes (3 espécies) e flutuantes fixas (1 espécie). As espécies mais frequentes foram Paspalum repens, Salvinia auriculata, Pistia stratiotes, Azolla sp., Lemna minor e Phyllanthus fluitans (Tabela 1).
Nos bancos de macrófitas aquáticas foram capturados 623 exemplares de peixes, distribuídos em 56 espécies, 37 gêneros, 15 famílias e 07 ordens (Tabela 2). A riqueza de espécies estimada pelo índice Jackknife, calculada para os lagos manejados foi 58,7 (dp 8,67), com intervalo de confiança entre 21,4 e $96(\mathrm{p}<0,05)$, sendo 25 o número máximo de ocorrência de espécies exclusivas. Para os lagos de preservaçáo foi 52 (dp 5,29), com intervalo de confiança entre 29,2 e $74,8(\mathrm{p}<0,05)$, sendo 24 o número máximo de ocorrência de espécies exclusivas.

Tabela 1 - Macrófitas aquáticas e capins flutuantes identificadas em seis lagos estudados na Amazônia Central, AM.

\begin{tabular}{|c|c|c|c|c|c|c|c|}
\hline \multirow{2}{*}{$\begin{array}{l}\text { Espécies } \\
\text { Azollaceae }\end{array}$} & \multicolumn{3}{|c|}{ Lagos de subsistência } & \multicolumn{3}{|c|}{ Lagos de preservação } & \multirow[t]{2}{*}{ Tipo ecológico } \\
\hline & Comandá & Praia & Araçá & Acari & Tracajá & Babaçú & \\
\hline Azolla sp. & $x$ & $\mathrm{X}$ & $x$ & $x$ & & $x$ & Flutuante \\
\hline \multicolumn{8}{|l|}{ Caratophyllaceae } \\
\hline Ceratopteris pteridoides Underw & & $\mathrm{X}$ & & & & & Flutuante \\
\hline \multicolumn{8}{|l|}{ Pontederiaceae } \\
\hline Eichhornia crassipes (Mar.) Salsus. & $\mathrm{x}$ & $x$ & $x$ & & $x$ & & Flutuante \\
\hline \multicolumn{8}{|l|}{ Poaceae } \\
\hline Hymenachne amplexicaule (Rudge.) Nees & & & & & $x$ & & Emergente \\
\hline Paspalum repens Berg & $x$ & $x$ & $x$ & & & $x$ & Emergente \\
\hline \multicolumn{8}{|l|}{ Lemnaceae } \\
\hline Lemna sp. & $x$ & & $x$ & $x$ & & $x$ & Flutuante \\
\hline \multicolumn{8}{|l|}{ Hydrocharitaceae } \\
\hline Limnobium sp. & & & & $x$ & & $x$ & Flutuante \\
\hline \multicolumn{8}{|l|}{ Onagraceae } \\
\hline Ludwigia natans Hum. \& Bonpl. & $x$ & & $x$ & $x$ & & & Flutuante \\
\hline \multicolumn{8}{|l|}{ Marsileaceae } \\
\hline Marsilea sp. & $x$ & & $x$ & & & $x$ & Flutuante \\
\hline \multicolumn{8}{|l|}{ Mimosaceae } \\
\hline Neptunia sp. & & & & $x$ & & $x$ & Flutuante \\
\hline \multicolumn{8}{|l|}{ Nymphaeaceae } \\
\hline Nymphaea sp. & & & $x$ & & & & Flutuante \\
\hline \multicolumn{8}{|l|}{ Euphorbiaceae } \\
\hline Phyllanthus fluitans Benth. Ex Müll. & $x$ & $x$ & $x$ & $x$ & & $x$ & \\
\hline \multicolumn{8}{|l|}{ Araceae } \\
\hline Pistia stratiotes L. & $x$ & $x$ & & $x$ & $x$ & $x$ & Flutuante \\
\hline \multicolumn{8}{|l|}{ Polygonaceae } \\
\hline Polygonum sp. & & & & & $x$ & & Emergente \\
\hline \multicolumn{8}{|l|}{ Ricciaceae } \\
\hline Ricciocarpus natans (L.) Corda & & & & $x$ & & & Flutuante \\
\hline \multicolumn{8}{|l|}{ Salviniaceae } \\
\hline Salvinia auriculata Aubl. & $x$ & $x$ & $x$ & $x$ & $x$ & $x$ & Flutuante \\
\hline \multicolumn{8}{|l|}{ Lentibulariaceae } \\
\hline Utricularia foliosa L. & & & $x$ & & & & Emergente \\
\hline
\end{tabular}


As curvas de acumulação (rarefação) para os lagos manejados e os de preservação mostraram um padrão crescente no número de espécies coletadas em função do número de exemplares amostrados, alcançando quase a assíntota na curva, tendendo a estabilização. Nos lagos de preservação, a curva possui menor inclinação sugerindo que a maioria das espécies foram coletadas (Figura 2).

Characiformes correspondeu a $65 \%$ da abundância total dos peixes capturados seguidos de Perciformes, $20,7 \%$, e Gymnotiformes, 9\%. Também foram capturados peixes das ordens Clupeiformes (3\%) Siluriformes (1\%),

Tabela 2 - Espécies de peixes e número de espécimes coletados nos bancos de macrófitas aquáticas e capins flutuantes em seis lagos na Amazônia Central AM. $M=$ espécies migratórias.

\begin{tabular}{|c|c|c|c|c|c|c|c|}
\hline \multirow{2}{*}{ Ordem, família, gênero e espécies } & \multirow{2}{*}{ Nome comum } & \multicolumn{3}{|c|}{ Lagos de subsistência } & \multicolumn{3}{|c|}{ Lagos de preservação } \\
\hline & & Comandá & Praia & Araçá & Acari & Tracajá & Babaçu \\
\hline \multicolumn{8}{|l|}{ Clupeiformes } \\
\hline \multicolumn{8}{|l|}{ Pristigateridade } \\
\hline Pellona castelnaeana Valenciennes, 1847 & apapá-amarelo & 2 & & & & & \\
\hline Pellona flavipinnis (Valenciennes, 1837) & apapá-branco, sardinhão & & 6 & & & & \\
\hline \multicolumn{8}{|l|}{ Engraulidae } \\
\hline Lycengraulis sp. & manjubinha & & 2 & 1 & & & 3 \\
\hline \multicolumn{8}{|l|}{ Osteoglossiformes } \\
\hline \multicolumn{8}{|l|}{ Arapaimatidae } \\
\hline Arapaima gigas (Schinz, 1822) & pirarucu & & 1 & & & & \\
\hline \multicolumn{8}{|l|}{ Characiformes } \\
\hline \multicolumn{8}{|l|}{ Erythrinidae } \\
\hline Hoplias malabaricus (Bloch, 1794) & traíra & 4 & 4 & 1 & 3 & 2 & 15 \\
\hline \multicolumn{8}{|l|}{ Hemiodontidae } \\
\hline Hemiodus sp. (M) & orana, charuto & & 4 & & & & \\
\hline \multicolumn{8}{|l|}{ Curimatidae } \\
\hline Potamorhina latior (Spix \& Agassiz, 1829)(M) & branquinha-comum & & 1 & & & & \\
\hline Potamorhina sp. (M) & branquinha & & & & 1 & & \\
\hline Curimata sp. (M) & branquinha & & & & 3 & & \\
\hline Curimata inornata Vari, 1989 (M) & branquinha & & & & & 1 & \\
\hline \multicolumn{8}{|l|}{ Characidae } \\
\hline Mylossoma duriventre (Cuvier, 1818) (M) & pacu-manteiga & 3 & 5 & & 1 & 2 & \\
\hline Mylossoma aureum (Spix \& Agassiz, 1829)(M) & pacu & & & & & & \\
\hline Serrasalmus rhombeus (Linnaeus, 1766) & piranha-preta & 1 & & 8 & 1 & & 8 \\
\hline Serrasalmus spilopleura Kner, 1858 & piranha-amarela & & 1 & & & 3 & \\
\hline Serrasalmus sp. & piranha & & & & & 1 & \\
\hline Metynnis argenteus Ahl, 1923 & pacu & & & & 2 & & 1 \\
\hline Roeboides myersii Gill, 1870 & zé-do-ó & & & & & & \\
\hline Ctenobrycon hauxwellianus (Cope, 1870) & piaba & & & 1 & & 2 & 4 \\
\hline Triportheus albus Cuvier, 1872 (M) & sardinha-comum & 24 & 17 & & & & \\
\hline Triportheus angulatus (Spix \& Agassiz, 1829)(M) & sardinha-papuda & 1 & & 1 & & 59 & \\
\hline Iguanodectes spilurus (Günther, 1864) & piaba & & & 1 & & & \\
\hline Hyphessobrycon copelandi Durbin, 1908 & piaba & & & 15 & & 1 & 6 \\
\hline Odontostilbe aff. madeirae Fowler, 1913 & piaba & & & 18 & & & 107 \\
\hline Odontostilbe sp. & piaba & 3 & 3 & & & & \\
\hline Hemigrammus sp. & piaba & 1 & 1 & & & & \\
\hline Hemigrammus analis Durbin, 1909 & piaba & & & 1 & & & \\
\hline
\end{tabular}


Tabela 2 - Continuação

\begin{tabular}{|c|c|c|c|c|c|c|c|}
\hline \multirow{2}{*}{ Ordem, família, gênero e espécies } & \multirow{2}{*}{ Nome comum } & \multicolumn{3}{|c|}{ Lagos de subsistência } & \multicolumn{3}{|c|}{ Lagos de preservação } \\
\hline & & Comandá & Praia & Araçá & Acari & Tracajá & Babaçu \\
\hline Hemigrammus bellottii (Steidachner,1882) & piaba & & & 1 & & & \\
\hline Hemigrammus schamardae (Steidachner, 1882) & piaba & & & & & & 1 \\
\hline Hemigrammus levis Durbin, 1908 & piaba & & & & & 7 & \\
\hline Moenkhausia collettii (Steidachner, 1882) & piaba & & & & & & 6 \\
\hline Moenkhausia lepidura (Kner, 1858) & piaba & 2 & & 13 & & & 9 \\
\hline Moenkhausia intermedia Eigenmann, 1908 & piaba & & 5 & 9 & & 3 & 1 \\
\hline Fluviphylax pygmaeus (Myers \& Carvalho, 1955) & piaba & & & 1 & & & \\
\hline Aphyocharax paraguayenses Eigenmann, 1915 & piaba & & 1 & & & & \\
\hline \multicolumn{8}{|l|}{ Cyprinodontiformes } \\
\hline \multicolumn{8}{|l|}{ Lebisianidae } \\
\hline Pyrhulina sp. & piaba & & & 1 & & & 1 \\
\hline Pyrhulina brevis Steindachner, 1876 & piaba & 2 & 1 & & & & \\
\hline \multicolumn{8}{|l|}{ Gymnotiformes } \\
\hline \multicolumn{8}{|l|}{ Sternopygidae } \\
\hline Eigenmannia virescens (Valenciennes, 1836) & peixe elétrico & & & 7 & & & \\
\hline Stenopygus macrurus (Bloch \& Schneider, 1801) & peixe elétrico & & & 1 & & & 1 \\
\hline \multicolumn{8}{|l|}{ Hipopomidae } \\
\hline Brachyhypopomus sp. & sarapó & 7 & 7 & 12 & 7 & 11 & \\
\hline \multicolumn{8}{|l|}{ Apteronotidae } \\
\hline Apteronotus sp. & peixe elétrico & & & 11 & & & \\
\hline \multicolumn{8}{|l|}{ Siluriformes } \\
\hline \multicolumn{8}{|l|}{ Auchenipteridae } \\
\hline Trachyleopterus galeatus (Linnaeus, 1766) & cangati & & & 1 & 1 & 3 & \\
\hline \multicolumn{8}{|l|}{ Callichthyidae } \\
\hline Hoplosternum littorale (Hancock, 1828) & tamoatá & & & & & 1 & \\
\hline \multicolumn{8}{|l|}{ Doradidae } \\
\hline Amblydoras hancockii (Valenciennes, 1840) & reco-reco & & & 1 & & & \\
\hline \multicolumn{8}{|l|}{ Perciformes } \\
\hline \multicolumn{8}{|l|}{ Cichlidae } \\
\hline Laetacara dorsigera (Heckel, 1840) & acará & 10 & 5 & 1 & & 4 & 4 \\
\hline Acaronia nassa (Heckel, 1840) & acará-cascudo & & 4 & 3 & & 2 & 4 \\
\hline Cichlasoma sp.1 & acará & & & & & 1 & \\
\hline Cichlasoma sp.2 & acará & & & & 2 & & \\
\hline Cichlasoma amazonarum Kullander, 1983 & acará & & 19 & & 12 & 1 & \\
\hline Mesonauta festivus (Heckel, 1840) & acará-boari & 1 & 6 & 7 & 3 & 3 & 7 \\
\hline Cichla sp. & tucunaré & & & 1 & & & \\
\hline Satanoperca jurupari (Heckel, 1840) & acará-bicudo & & & & & 1 & \\
\hline Apistogramma agassizii (Steindachner, 1875) & acará & & 4 & & & & \\
\hline Apistogramma geisleri Meinken, 1971 & acará & & & & & 3 & \\
\hline Apistogramma sp. & acará & & & 4 & & 2 & \\
\hline Taeniacara candidi Myers, 1935 & acará & 2 & 2 & & & & \\
\hline \multicolumn{8}{|l|}{ Synbranchiformes } \\
\hline \multicolumn{8}{|l|}{ Synbranchidae } \\
\hline Synbranchus marmoratus Bloch, 1975 & mussum & & 1 & 1 & & 2 & \\
\hline
\end{tabular}




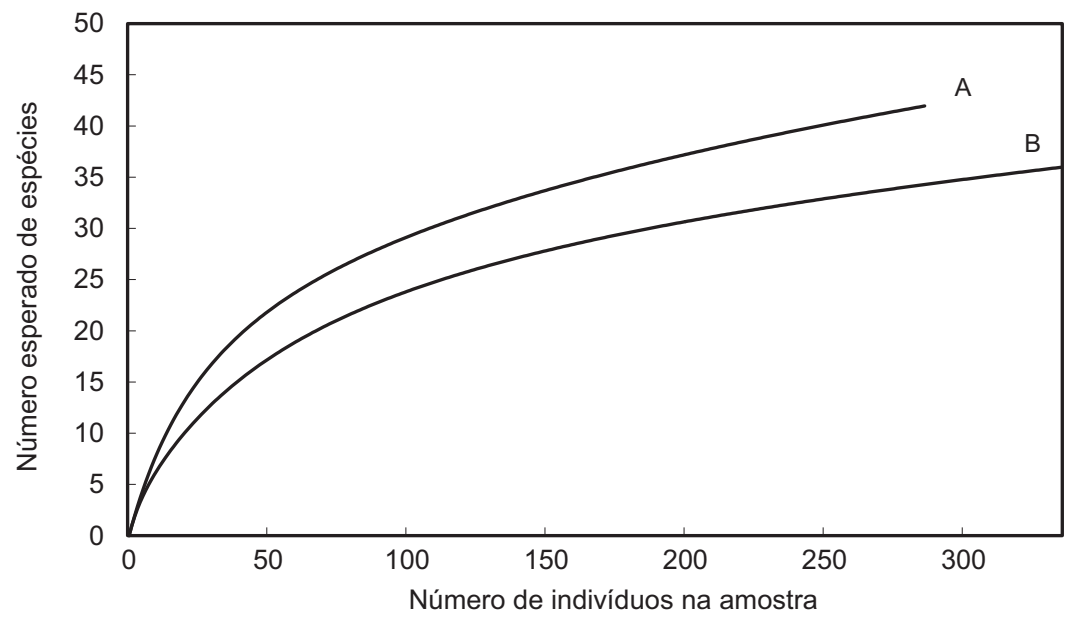

Figura 2 - Curva de rarefação das assembleias de peixes dos lagos manejados para subsistência (A) e mantidos para a preservação (B), capturadas aos bancos de macrófitas aquáticas e capins flutuantes na Amazônia Central, AM.

Tabela 3 - Atributos das assembleias de peixes associadas aos bancos de macrófitas aquáticas e capins flutuantes em seis lagos estudados na Amazônia Central, AM.

\begin{tabular}{lcccccc}
\hline & \multicolumn{3}{c}{ Lagos } \\
\cline { 2 - 6 } \multicolumn{1}{c}{ Atributos ecológicos } & \multicolumn{4}{c}{ De manejo (subsistência) } & \multicolumn{2}{c}{ Preservação } \\
\cline { 2 - 6 } & Comandá & Praia & Araçá & Acari & Tracajá & Babaçu \\
\hline Número de exemplares (N) & 63 & 100 & 124 & 34 & 123 & 179 \\
Riqueza (S) & 14 & 22 & 27 & 10 & 23 & 17 \\
Peso total (g) & 287,56 & 376,91 & 152,04 & 57,5 & 653 & 119 \\
Shannon-Weaver (H') & 2,99 & 3,88 & 3,97 & 2,77 & 3,1 & 2,39 \\
\hline Equitabilidade (E) & 0,79 & 0,87 & 0,83 & 0,83 & 0,69 & 0,58 \\
Berger-Parker (1/d) & 2,63 & 5,26 & 6,67 & 2,83 & 2,08 & 1,67 \\
Dominância (d) & 0,38 & 0,19 & 0,15 & 0,35 & 0,48 & 0,60 \\
\hline
\end{tabular}

Synbranchiformes $(0,7 \%)$ e Osteoglossiformes $(0,2 \%)$. Characidae foi a família mais abundante seguida de Cichlidae, Hypopomidae, Curimatidae e Erythrinidae. Odontostilbe aff. madeira, Triportheus angulatus, Brachyhypopomus sp. T. albus e Cichlasoma amazonarum tiveram os maiores números de exemplares, ao passo que Mesonauta festivus e Hoplias malabaricus as maiores frequências de ocorrência (Tabela 2). H. malabaricus, Moenkhausia intermedia, Brachyhypopomus sp., Laetacara dorsiger, M. festivus foram capturadas em quase todos os lagos. T. albus predominou no Comandá, $C$. amazonarum e $T$. albus no Praia, T. angulatus no Tracajá e Odontostilbe aff. madeira, $M$. lepidura, $H$. copelandi no Araçá e Babaçu (Figura 3).

Nos lagos Tracajá, Praia e Babaçu foram capturados os maiores números de exemplares, mas o maior peso total foi obtido nas coletas efetuadas nos lagos Tracajá e Praia. A riqueza e a diversidade de Shannon-Weaver foram menores no Comandá, Acari, Babaçu e maiores no Praia e Araçá e Tracajá. Os valores do inverso do Índice de dominância de BergerParker foram maiores no Praia e Araçá, lagos com menores valores de dominância. A equitabilidade foi baixa no Babaçu e Tracajá indicando a maior dominância de espécies (Tabela 3).

A análise de grupamento (Cluster) dos índices de similaridade (Bray-Curtis) estimados para as amostragem dos peixes separou os lagos com a formação de três grupos. O primeiro "A" é composto por dois subgrupos, um formado por Praia (PRAm) e Comandá (COMm), e o outro por Acari (ACAp), todos próximos ao rio Amazonas. O segundo " $\mathrm{B}$ " contém o Tracajá (TRAp), também próximo ao rio, e por último, o "C" com Araçá (ARAm) e Babaçu (BABp), os mais distantes do rio (Figura 4).

A análise de covariância indica que tipo de uso, subsistência e preservação, e distância do lago em relação ao rio não influenciaram significativamente nas variáveis resposta, abundância, riqueza, peso total, diversidade de Shannon-Weaver, diversidade Berger-Parker, equitabilidade e dominância (Tabela 4). E, a composição de espécies entre os lagos também não foi significantemente diferente, sendo comprovada pelo resultado da análise de similaridade (ANOSIM R = -0,296; $\mathrm{p}=1 ; 999$ permutaçóes). 


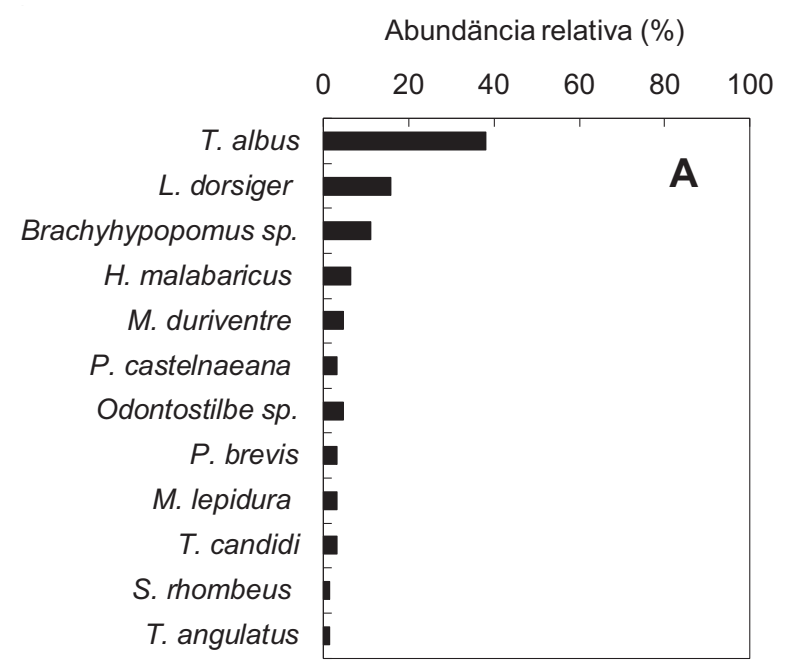

Abundância relativa (\%)

$\begin{array}{llllll}0 & 20 & 40 & 60 & 80 & 100\end{array}$
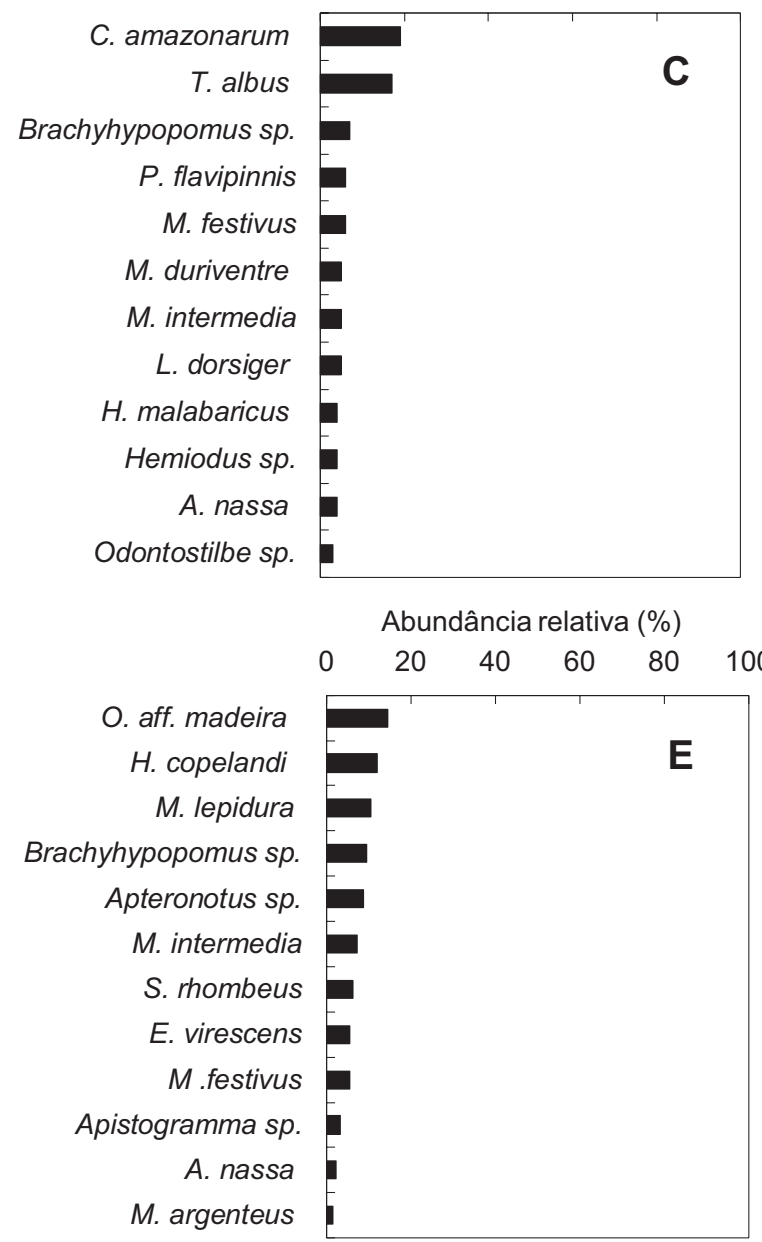

Abundäncia relativa (\%)

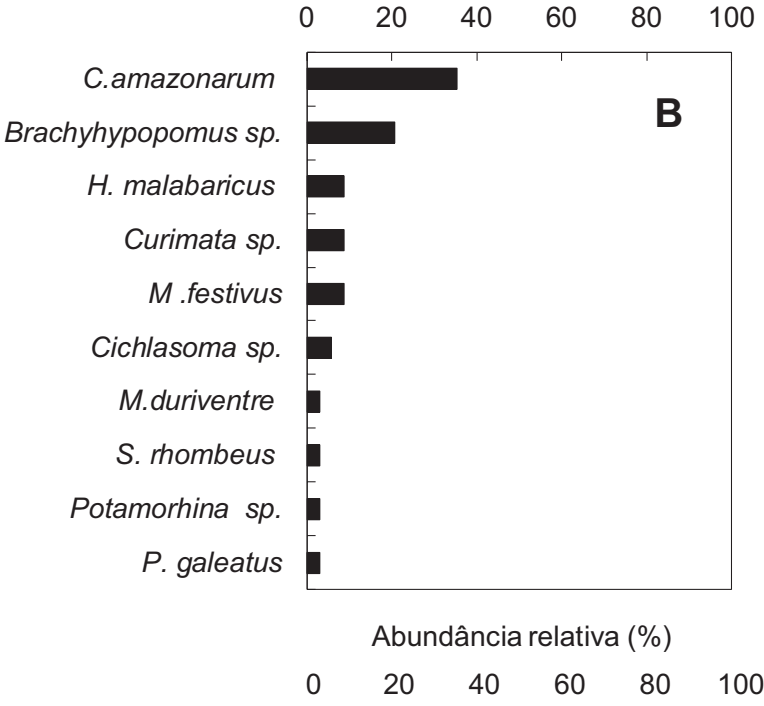

$$
\begin{array}{r}
\text { T. angulatus } \\
\text { Brachyhypopomus sp. } \\
\text { R. myersii } \\
\text { H. levis } \\
\text { L. dorsiger } \\
\text { S. spilopleura } \\
\text { M. intermedia } \\
\text { P. galeatus } \\
\text { A. geisleri } \\
\text { M. festivus } \\
\text { H. malabaricus } \\
\text { M. duriventre }
\end{array}
$$

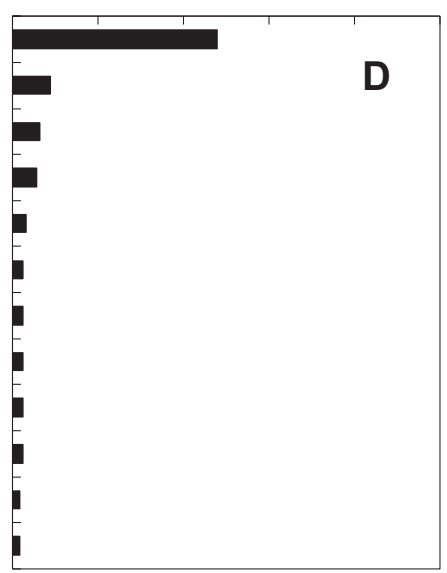

Abundäncia relativa (\%)

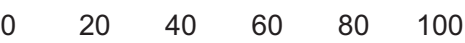

\section{O. aff. madeira}

H. malabaricus

M. lepidura

S. rhombeus

M. festivus

H. copelandi

C. hauxwellianus

L. dorsiger

A. nassa

M. collettii

Lycengraulis sp.

M. aureum

Figura 3 - Composição das espécies de peixes mais abundantes coletadas nos bancos de macrófitas aquáticas e capins flutuantes em seis lagos na Amazônia Central, AM. Os painéis A: Comandá, C: Praia e E: Araçá, correspondem aos lagos manejados para a subsistência. Os painéis B: Acari, D: Tracajá e F: Babaçu correspondem aos lagos de preservação. 


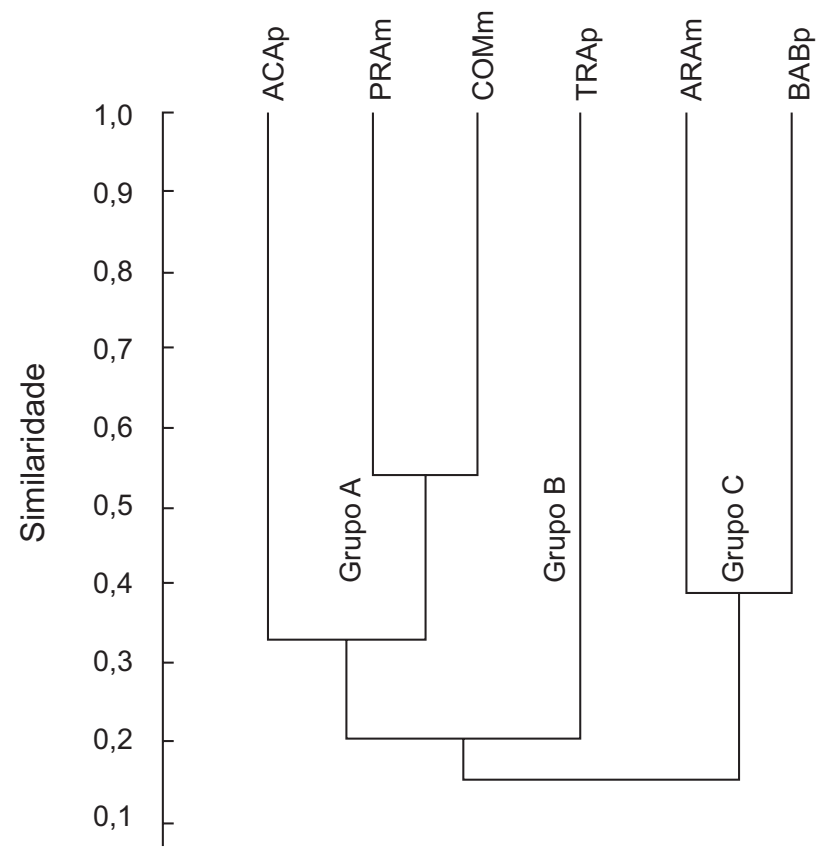

Figura 4 - Dendrograma gerado a partir do índice de similaridade de Bray Curtis entre seis os lagos amostrados na Amazônia Central, AM. TRAp= Tracajá (preservação), $\mathrm{COMm}=$ Comandá (manejo), $\mathrm{ACAp}=$ Acari (preservação), PRAm $=$ Praia $($ manejo $), A R A p=$ Araçá $($ manejo $), B A B p=$ Babaçu (preservação).

\section{DISCUSSÃO}

A ordem Characiformes tem sido relatada como a ordem dominante em lagos de várzea, tanto na floresta alagada (SaintPaul et al. 2000), água aberta (Saint-Paul et al. 2000) como nos bancos de macrófitas aquáticas (Sánchez-Botero e AraújoLima 2001; Prado et al. 2009) da Amazônia. A predominância em termos de riqueza e em número de indivíduos também foi identificada nos bancos de macrófitas aquáticas dos lagos Comandá, Praia, Araçá, Acari, Tracajá e Babaçu.

A estimativa Jackknife de riqueza de espécies calcula que acima de $50 \%$ das espécies foram capturadas nos lagos, manejados e de preservação , sugerindo assim, que a ictiofauna foi bem amostrada. A curva de rarefação mostra que o esforço amostral empregado nos lagos foi suficiente para amostrar a ictiofauna local, ou seja, o número de espécies coletadas foi muito próximo do existente na área.

A captura de 56 espécies é similar a outras efetuadas em lagos no médio Amazonas, 50 espécies (Prado et al. 2010) e na reserva de Mamirauá, 79 espécies (Henderson e Hamilton 1995). Mas é diferente do número de peixes capturados nas macrófitas de lagos próximos a Manaus, Janauacá, Camaleão e do Rei, 91 a 180 espécies (Sánchez-Botero e Araújo-Lima 2001). Isso pode ser explicado por diferenças metodológicas, especialmente pelo grande número de amostragens efetuadas nesse lagos.
A ictiofauna associada aos bancos de macrófitas aquáticas capturada nos lagos Comandá, Praia, Araçá, Acari, Tracajá e Babaçu, foi bem amostrada até a profundidade de $5 \mathrm{~m}$. Dentro dessa faixa de profundidade foram coletadas espécies já amplamente relatadas nos lagos de várzea (Araújo-Lima et al. 1986; Prado et al. 2010) como C. hauxwellianus, Hemigrammus spp., Hyphessobrycon spp., Moenkhauasia spp., Odontostilbe spp., M. festivus, C. amazonarum e Apistogramma spp., espécie de pequeno porte, e $S$. spilopleura, $H$. malabaricus, S. marmoratus, Brachyhypopomus sp., Eigenmannia sp., Apteronotus sp., M. duriventre, T. albus e T. angulatus, espécie de médio porte. As migradoras de importância comercial, aquelas que realizam deslocamentos periódicos entre a área alagada e rio (Fernandes 1997; Winemiller e Jepsen 1998), tais como Colossoma macropomum, Rythiodus spp., Semaprochilodus spp., Prochilodus nigricans e Brycon spp., comuns nos bancos de macrófitas (Sánchez-Botero e Araújo-Lima 2001; Petry et al. 2003; Correa et al. 2008), não foram capturadas nos seis lagos. Essa ausência pode ser explicada pela reduzida abundância dessas espécies na regiâo pelágica desses lagos (obs. pessoal).

Não foi observado efeito do tipo de uso, subsistência e preservação, e da distância do lago em relação ao rio, nos atributos ecológicos das assembleias de peixes (abundância, riqueza, peso total, diversidade de Shannon-Weaver, diversidade Berger-Parker, equitabilidade e dominância). Tampouco a composição de espécies variou entre os tipos de lagos. A ausência de efeito ou a não detecção do efeito do tipo do manejo nas análises das assembleias de peixes associadas aos bancos de macrófitas aquáticas e capins flutuantes, pode ser explicada por diversos fatores: (i) pouco tempo de manejo efetivo, cerca de 5 anos; (ii) o baixo número de pessoas por família que explota a ictiofauna nos lagos de subsistência; (iii) a agricultura ser a principal atividade econômica de subsistência, resultando em reduzido esforço de pesca; (iv) inexistência de pescarias em larga escala que produzam alteraçóes ambientais significativas; e (v) a existência de um fator ecológico de grande intensidade, o pulso de inundação, que se sobrepóe a outros efeitos de menor intensidade.

\section{AGRADECIMENTOS}

Os autores são gratos pelo apoio financeiro ao $\mathrm{CNPq}$ (PNOPG nº1161/99), FAPEAM (nº189/03) e à Financiadora de Estudos e Projetos - FINEP e a Petróleo Brasileiro SA - PETROBRAS, por meio do projeto PIATAM. Também agradecemos a Jansen Zuanon e Hélio D.B. dos Anjos pelo auxílio na identificaçáo dos peixes e aos avaliadores pelas sugestôes que melhoraram a qualidade do manuscrito. 


\section{BIBLIOGRAFIA CITADA}

Araújo-Lima, C.A.R.M.; Portugal, L.P.S.; Ferreira, E.G. 1986. Fish-macrophytes relationship in the Anavilhanas Archipelago, a black water system in the Central Amazon. Journal of Fish Biology, 29: 1-11.

Berger, W.H.; Parker, F.L. 1970. Diversity of planktonic foraminifera in deep sea sediments. Science, 68: 1345-1347.

Bulla, C.K.; Gomes, L.C.; Miranda, L.E.; Agostinho, A.A. 2011. The ichthyofauna of drifting macrophyte mats in the Ivinhema River, upper Paraná River basin, Brazil. Neotropical Ichthyology, 9: 403-409.

Clarke, K.R. 1993. Non-parametric multivariate analyses of changes in community structure. Australian Journal of Ecology, 18: $117-143$

Correa, S.B.; Crampton, W.G.R.; Chapman, L.J.; Albert, J.S. 2008. A comparison of flooded and floating meadow fish assemblages in a upper Amazon floodplain. Journal of Fish Biology, 72: 629-644.

Fernandes, C.C. 1997. Lateral migration of fishes in Amazon floodplains. Ecology of Freshwater Fish, 6: 36-44.

Géry, J. 1977. Characoids of the world. TFH publications, Neptune City, USA. 1977, 672p.

Goulding, M. 1997. História Natural dos Rios Amazônicos. Trad. Antônio Carlos de A. dos Santos e Mírian Leal Carvalho. Sociedade Civil Mamirauá/CNPq, Brasília, Rainforest Alliance, 1997, 208p.

Heltshe, J.; Forrester, N. 1983. Estimating species richness using the Jackknife procedure. Biometrics, 39: 1-11.

Henderson, P.A.; Hamilton, H.F. 1995. Standing crop and distributions of fish in drifting and attached floating meadow within and Upper Amazonian varzea lake. Journal of Fish Biology, 47: 266-276.

Junk, W.J. 1973. Investigations of the ecology and prodution-biology of the "floating meadows" (Paspalo-Echinochloetum) on the Midlle Amazon. II. The aquatic fauna in the root zone of floating vegetation. Amazoniana, 4: 9-112.

Krebs, C.J. 1989. Ecological methodology. Harper \& Row Publishing, New York, 1989, 653p.

Kullander, S.O. 1986. Cichlid Fishes of the Amazon River Drainage of Peru. Monograph. Museum of Natural History, Stockholm, Sweden, 1986, 434p.

Lopes, A.; Paula, J.D. ; Mardegan, S.F.; Hamada, N.; Piedade, M.T.F. 2011. Influência do hábitat na estrutura da comunidade de macroinvertebrados aquáticos associados às raízes de Eichhornia crassipes na região do Lago Catalão, Amazonas, Brasil. Acta Amazonica, 41: 493-502
Mago-Leccia, F. 1994. Electric fishes of the continental waters of America. Fundacion para el Desarrollo de las Ciencias Fisicas, Matemáticas y Naturales, Caracas, Venezuela, 1998, 223p.

Planquette, P.; Keith, P.; Le Bail, P.Y. 1996. Atlas des poissons d'eau douce de Guyane. Collection du Patrimoine Naturel, v. 22. Paris, 1996, 429p.

Petry, P.; Bayley, P.B.; Markle, D.F. 2003. Relationships between fish assemblages, macrophytes and environmental gradients in the Amazon River floodplain. Journal of Fish Biology, 63: 547-579.

Prado, K.L.L.; Freitas, C.E.C.; Soares, M.G.M. 2009. Assembleias de peixes associadas a diferentes bancos de macrófitas aquáticas em lagos de várzea do baixo rio Solimóes. Revista Colombiana de Ciencia Animal, 1: 185-201.

Prado, K.L.L.; Freitas, C.E.C.; Soares, M.G.M. 2010. Assembleias de peixes associadas às macrófitas aquáticas em lagos de várzea do baixo rio Solimóes. Revista Biotemas, 23: 131-142.

Rubim, A. 2001. Estudos ecológicos de duas espécies de macrófitas aquáticas, Salvinia molesta Mitchell e Pistia stratiotes L: Taxa de crescimento e ciclagem de nutrientes. Tese de Doutorado, Universidade Estadual Paulista, Rio Claro, São Paulo. 121p.

Saint-Paul, U.; Zuanon, J.; Villacorta Correa, M.A.; Garcia, M.; Fabré, N.N.; Berger, U.; Junk, W.J. 2000. Fish communities in central Amazonian white- and blackwater floodplains. Environmental Biology of Fishes, 57: 235-250.

Sánchez-Botero, J.I.; Araújo-Lima, C.A.R.M. 2001. As macrófitas aquáticas como berçário para a ictiofauna da várzea do rio Amazonas. Acta Amazonica, 31: 437-447.

Sánchez-Botero, J.I.; Farias, M.L.de; Piedade, M.T.; Garcez, D.S. 2003. Ictiofauna associada às macrófitas aquáticas Eichhornia azurea (SW.) Kunth. e Eichhornia crassipe. Acta Scientiarum Biological Sciences, 25: 369-375.

Schiesari, L.; Zuanon, J.; Azevedo-Ramos, C.; Garcia, M.; Gordo, M.; Messias, M.; Monteiro, E. 2003. Macrophyte rafts as dispersal vectors for fishes and amphibians in the Lower Solimóes River, Central Amazon. Journal of Tropical Ecology, 19: 333-336.

Winemiller, K.O.; Jepsen, D.B. 1998. Effects of seasonality and fish movement on tropical river food webs. Journal of Fish Biology, 53: $267-296$

Zar, J.H., 1996. Biostatistical Analysis. 3rd Edn. Prentice Hall Inc., Englewood Cliffs, New Jersey, USA, 1996, 662p.

Recebido em 25/09/2012

Aceito em 21/03/2013 